\title{
LITÍASE RENAL NO PRÉ E PÓS-TRANSPLANTE RENAL
}

\author{
Urinary Calculi in Renal Transplant Recipients and in Renal Donors
}

Érika Bevilaqua Rangel', Samirah Abreu Gomes', Ita Pfeferman Heilberg²

\section{RESUMO}

A incidência de litíase renal na população geral vem aumentando e esta é uma contra-indicação relativa para a doação renal. A avaliação do potencial doador renal inclui exames laboratoriais e de imagem, podendo o doador ser liberado, mesmo quando há história pregressa de litíase renal, na ausência de distúrbio metabólico (hipercalciúria, hiperoxalúria, hiperuricosúria e/ou hipocitratúria, acidose metabólica), na ausência de história de cálculo de cistina ou estruvita, na ausência de infecção urinária e na ausência de múltiplos cálculos ou nefrocalcinose. Os cálculos renais presentes em doadores falecidos ou vivos podem ser eliminados espontaneamente após o transplante ou podem ser removidos pela ureteroscopia, nefrolitotomia percutânea ou litotripsia extracorpórea. A litíase renal após o transplante renal é um evento raro e pode acontecer tanto de novo, quanto já estar presente no rim do doador, com incidência variando de 0,2 a 3\%. Os pacientes são usualmente assintomáticos, já que o rim transplantado é denervado. O diagnóstico pode ser feito em três meses a três anos após o transplante, com maior freqüência no primeiro ano. A composição dos cálculos difere da população geral, de modo que os cálculos de ácido úrico ou aqueles associados à infecção urinária são mais freqüentes. O tratamento depende do tamanho do cálculo, da sua localização e da experiência do centro transplantador, incluindo a litotripsia extracorpórea, a nefrolitotomia percutânea ou aberta, ureterorenoscopia, a cistoscopia e a observação clínica (eliminação espontânea).

Descritores: Litíase Renal, Transplante Renal, Tratamento, Doadores de Órgãos.

Grau Acadêmico:

1. Doutoranda da Disciplina de Nefrologia

2. Doutoranda da Disciplina de Nefrologia

3. Professora Adjunta da Disciplina de Nefrologia

Instituição:

UNIFESP - Universidade Federal de São Paulo - Disciplina de Nefrologia

Correspondência:

Érika Bevilaqua Rangel

Rua Botucatu, 740 - CEP 04023-900 - São Paulo - SP - Brasil

Tel: 551155746300 / Fax: 551155739652

E-mail: erikabr@uol.com.br

Recebido em: 31/01/2006

\section{INTRODUÇÃO}

A incidência de litíase na população geral vem crescendo, atingindo até $12 \%$ da população geral americana e com uma taxa de recorrência em torno de $50 \%$ em cinco anos e de $75 \%$ em 20 anos. ${ }^{1,2}$

\section{AVALIAÇÃO DO DOADOR COM LITÍASE RENAL}

Na avaliação do doador renal com história pregressa de litíase renal, a anamnese deve incluir informações de história familiar de litíase renal, bem como de casamentos consangüíneos, para auxiliar na identificação de doenças de base, como cistinúria, hiperoxalúria primária, acidose tubular renal primária e nefrocalcinose.

Os exames laboratoriais para investigação do doador renal com história pregressa de litíase renal devem incluir:

a) exames séricos: creatinina, cálcio, fósforo, paratormônio, ácido úrico, potássio e gasometria venosa;

b) sedimento urinário

c) $\mathrm{pH}$ urinário de jejum de 12 horas

c) urina de 24 horas: creatinina, cálcio, ácido úrico, citrato, oxalato, sódio, magnésio e cistina.

Além dos exames laboratoriais, a avaliação radiológica para investigação de litíase renal deve incluir o raio $\mathrm{X}$ simples de abdome para detecção de cálculos radiopacos, o ultra-som renal (US), a urografia excretora (UGE) e a tomografia helicoidal de rins e vias urinárias (TC). A UGE é mais importante para a verificação de alterações anatômicas do trato urinário e a TC é melhor para a identificação de litíase. 
O US renal tem sensibilidade de 45 a $74 \%$ e especificidade em torno de $77 \%$ para o diagnóstico de litíase renal ${ }^{3,4}$ e pode, mais raramente, revelar a presença de cálculos em pacientes assintomáticos, o que ocorre em 0,4 a $0,64 \%$ dos casos. ${ }^{5}$

A TC helicoidal tem sensibilidade de 94 a $100 \%$ e especificidade de $100 \%$ para o diagnóstico de litíase renal ${ }^{1,3,4}$ comparado com sensibilidade de 66 a $87 \%$ e especificidade de $94 \%$ para a UGE. ${ }^{1,4}$ Os valores preditivos positivo e negativo de diagnóstico de litíase renal para a TC helicoidal são de $100 \%$ e $91 \%$, respectivamente, enquanto que para a UGE são de $97 \%$ e $74 \%$, respectivamente. ${ }^{1}$

A litíase renal é uma contra-indicação relativa para a doação renal, já que é possível estabelecer o risco anual de recorrência de cálculos no rim remanescente $\left(0,5\right.$ e 0,4 para homens e mulheres, respectivamente). ${ }^{6}$ Entretanto, $49 \%$ dos centros franceses de transplante recusam doadores com história pregressa de litíase renal.?

Um potencial doador renal assintomático, com história pregressa de cálculo único, pode ser liberado para a doação renal, se apresentar: ${ }^{8}$

1. Ausência de distúrbio metabólico (hipercalciúria, hiperoxalúria, hiperuricosúria e/ou hipocitratúria, acidose metabólica).

2. Ausência de história de cálculo de cistina ou estruvita. Os cálculos de estruvita representam cerca de $15 \%$ dos cálculos e estão associados à infecção urinária.

3. Ausência de infecção urinária.

4. Ausência de múltiplos cálculos ou nefrocalcinose na TC atual.

Se houver diagnóstico de cálculo renal atual, o doador renal pode ser liberado se: ${ }^{8}$

1. O doador preencher os primeiros três critérios anteriores.

2. Cálculos $<1.5 \mathrm{~cm}$ ou passíveis de serem removidos durante o transplante.

As contra-indicações absolutas para a doação renal incluem nefrocalcinose, cálculos bilaterais ou cálculos com alta taxa de recorrência: cistina, estruvita, cálculos associados com distúrbios sistêmicos ou hereditários (hiperoxalúria primária ou entérica, acidose tubular renal distal, sarcoidose) e cálculos associados à doença inflamatória intestinal. ${ }^{8}$

No entanto, existem vários relatos de doadores vivos ${ }^{5,9,10-12} \mathrm{e}$ falecidos, ${ }^{5,9,12-16}$ com litíase renal diagnosticada previamente à doação ou com diagnóstico no intra-operatório. Quando o cálculo está presente no cálice do rim extirpado para doação, sugere-se não realizar o tratamento na mesa cirúrgica, já que a pielolitotomia se associa a maior risco de fístula urinária. ${ }^{5} \mathrm{Na}$ maior parte das vezes, ocorre eliminação espontânea do cálculo, embora sua permanência esteja associada à fístula urinária, à infecção urinária e à uretero-hidronefrose. ${ }^{5}$ Quando o cálculo não é eliminado, é recomendada a ureteroscopia, nefrolitotomia percutânea ou litotripsia extracorpórea (LECO). Por outro lado, no caso de cálculos ureterais, estes devem ser sempre removidos no período pré-operatório. ${ }^{5}$ Contudo, a melhor opção de tratamento para os casos de litíase no rim do doador permanece controversa, já que existem poucos casos descritos na literatura.

Em doadores renais vivos com litíase renal incidental diagnosticada por TC, o tratamento do cálculo pode ser realizado antes ou depois do transplante. ${ }^{10}$ No caso da LECO realizada antes do transplante, recomenda-se aguardar um período de pelo menos seis semanas para realizar a nefrectomia. ${ }^{10} \mathrm{~A}$ LECO realizada em um doador vivo com cálculo de oxalato de cálcio permitiu a eliminação completa do cálculo e possibilitou a doação renal após três meses, sem recorrência no doador. ${ }^{12}$ Em outro doador renal com dois cálculos de ácido úrico de 5 e 6 mm, a LECO levou à eliminação parcial dos cálculos, sendo os três fragmentos remanescentes retirados por pielotomia no intraoperatório. ${ }^{12}$ Neste último caso, também não houve recorrência da litíase renal, nem infecção urinária.

Por outro lado, a ureteroscopia ex vivo realizada em solução salina em dez doadores vivos, mostrou-se ser também um procedimento seguro e eficaz para a remoção de cálculos unilaterais pequenos e não obstrutivos, com tamanho de 1 a $8 \mathrm{~mm} .{ }^{11}$ A ureteroscopia ex vivo manteve a integridade do ureter transplantado e não influenciou a função renal. Além disto, após um tempo de seguimento aproximado de 33 meses, nenhum dos receptores formou um novo cálculo no rim transplantado e nenhum doador teve recorrência da litíase renal.

Doadores falecidos geralmente não são submetidos a US antes da nefrectomia, o que resulta em maior possibilidade de se transplantar um rim com cálculos.

O US teve sensibilidade em torno de $40 \%$ em detectar litíase renal em doadores cadavéricos previamente à nefrectomia, o que possibilitou a remoção endoscópica por pielotomia no intraoperatório em três casos e a não utilização do rim em um caso em que havia pus associado à presença do cálculo. ${ }^{9}$ Em nenhum dos casos houve recorrência da litíase. Em outros quatro doadores falecidos com cinco cálculos renais variando de 4 a $15 \mathrm{~mm}$ não detectados ao US prévio, a abordagem dos cálculos ocorreu no período pós-operatório com a utilização da LECO, embora tenha sido relatada também eliminação espontânea dos cálculos. ${ }^{9}$ Em três pacientes que receberam rim de doador falecido com litíase renal, a nefrolitotomia percutânea foi realizada com sucesso um a dois meses após o transplante. ${ }^{13}$ Por outro lado, um outro caso de um paciente que recebeu um rim de doador falecido com cálculo de oxalato de cálcio, a evolução foi menos favorável. ${ }^{12}$ Após a LECO, houve obstrução ureteral, hidronefrose e oligúria, sendo o cálculo removido por cistoscopia e realizado implante de cateter duplo J.

Em resumo, a anamnese detalhada, a realização de exames laboratoriais e de imagem e a checagem das contra-indicações para a doação renal devem ser analisadas nos casos de candidatos à doação renal com história pregressa de litíase renal.

\section{LITÍASE RENAL NO RIM TRANSPLANTADO}

As complicações urológicas após o transplante renal são descritas em 7,1 a 9,2\% dos $\operatorname{casos}^{17-19}$, sendo as mais freqüentes as fístulas urinárias e as obstruções ureterais primárias por isquemia ou por falha técnica ${ }^{17-19}$. A litíase renal após o transplante renal é um evento raro e pode acontecer tanto de novo, quanto já estar presente no rim do doador. A incidência varia de 0,2 a $3 \%^{9,12,15-31}$, sendo em média $1 \%$. Os dados do USRDS (USA Renal Data System) indicam incidência menor de $0,11 \%$ para homens e de $0,15 \%$ para mulheres, embora esta diferença entre os sexos não seja significativa. ${ }^{21}$ Não há correlação, portanto, entre a idade, sexo ou raça do doador ou do receptor, tempo de isquemia fria ou implante ureteral com a ocorrência de litíase, após o transplante renal. ${ }^{21,24}$

A incidência de litíase renal na população de transplantados com doadores vivos é menor do que na população geral, uma vez que estes doadores realizam exames de imagem previamente à doação para descartar a presença de litíase renal. As mulheres transplantadas 
renais teriam uma maior incidência de litíase renal do que as mulheres na população geral, por razões ainda não esclarecidas. ${ }^{21}$

Usualmente, os pacientes submetidos ao transplante renal e que apresentam litíase são assintomáticos ${ }^{24,31}$, de modo que muitos casos não são diagnosticados. Geralmente, os cálculos são diagnosticados de três meses a três anos e meio após o transplante, com maior freqüência no primeiro ano pós-transplante. ${ }^{19,24,32} \mathrm{O}$ tempo de internação do paciente com litíase renal após o transplante é de três dias em média, sendo que o risco do paciente transplantado renal se internar com litíase renal é maior do que na população geral. ${ }^{21}$

O quadro clínico mais comum é a piora da função renal, podendo chegar a anúria se a obstrução ureteral for completa. ${ }^{24,33}$ Os sintomas relacionados à obstrução e, portanto, à cólica nefrética, geralmente estão ausentes, já que o ureter do rim transplantado é denervado. ${ }^{5,10,13,22,31,33}$ Contudo, algumas vezes, pode estar presente um certo grau de desconforto na fossa ilíaca onde o rim está transplantado, além de massa palpável, o que é atribuído à distensão do rim acompanhada do estiramento da fáscia e da musculatura da parede abdominal, o que pode simular um quadro de rejeição aguda. ${ }^{33}$ Hematúria assintomática pode também estar presente. ${ }^{22,26}$

$\mathrm{O}$ aparecimento de litíase de novo está relacionado a alterações metabólicas, infecção urinária ou presença de um corpo estranho na via urinária, como um cateter ureteral duplo $\mathrm{J}$ ou fio de sutura nãoabsorvível, além de refluxo vésico-ureteral. ${ }^{22,26,33}$ A infecção urinária pode ser causa ou conseqüência da litíase renal. . $2,33^{2}$

As alterações metabólicas podem estar presentes isoladamente ou associadas, sendo as mais freqüentes: hiperparatiroidismo terciário, hipercalciúria, hipocitratúria e hiperuricosúria. ${ }^{22,24,27}$ Como a formação dos cálculos é de etiologia multifatorial, as alterações metabólicas podem estar também associadas a alterações anatômicas, a obstrução ureteral e/ou a infecção urinária recorrente..$^{15,32}$

Por outro lado, os pacientes transplantados renais em uso de ciclosporina, azatioprina e prednisona em doses reduzidas (5 a 10 $\mathrm{mg} /$ dia) não apresentariam maior risco de formação de cálculos de oxalato de cálcio. ${ }^{34}$

A recorrência da litíase renal é descrita em cerca de 17 a $30 \%$ dos pacientes transplantados renais ${ }^{9,15,16,22,24,26}$, podendo ocorrer perda do enxerto renal decorrente do cálculo renal em 6 a $11,1 \%{ }^{15,17}$ Assim, a litíase no rim transplantado não parece ser uma causa que compromete de modo importante o funcionamento do enxerto renal a longo prazo, ${ }^{21,32}$ nem está associada à maior mortalidade. ${ }^{21}$

Embora os pacientes submetidos ao transplante duplo de pâncreasrim tenham maior tendência de apresentar litíase renal em relação ao transplante renal, a incidência de litíase não difere significantemente naquelas duas modalidades de transplante (duplo pâncreas-rim $1,2 \%$ e rim $0,23 \%) .{ }^{23}$

$\mathrm{O}$ diagnóstico da litíase após o transplante renal deve incluir o raio-X de abdome, US renal, a TC renal e os exames laboratoriais de sangue e urina. ${ }^{26} \mathrm{O}$ US é uma ferramenta útil para determinar a localização e o tamanho dos cálculos no rim transplantado, além de mostrar a hidronefrose. ${ }^{22} \mathrm{~A}$ TC renal é mais precisa para a determinação exata do cálculo, especialmente quando um procedimento cirúrgico é programado. ${ }^{14}$

A localização dos cálculos no rim transplantado é variável na literatura: calicial 20 a $75 \%$ 9,16,19,21-23, ureteral em 12,5 a 75\% 9,16,17,19,21,23 e vesical em 18,2 a $50 \%$ 9,16,19,23 ou múltiplos sítios em 6,7 a $43,8 \%$ dos casos. ${ }^{15,22}$

Tabela 1 - Relatos de litíase renal após o tratamento renal na literatura

\begin{tabular}{|c|c|c|c|c|c|c|c|c|}
\hline Referências & {$[26]$} & {$[23]$} & [22] & {$[15]$} & [9] & {$[17]$} & [21] & [24] \\
\hline Número de pacientes & 1500 & 1813 & 849 & 794 & 1027 & 1535 & 39628 & 1500 \\
\hline Incidência litíase & $0,7 \%$ & $0,44 \%$ & $1,8 \%$ & $2 \%$ & $1,6 \% \gamma$ & $0,78 \%$ & $0,13 \%$ & $0,8 \%$ \\
\hline $\begin{array}{l}\text { Tempo médio de diagnóstico } \\
\text { (dias, meses ou anos) }\end{array}$ & & 68,8 meses & 17,8 meses (3-109) & & 28 meses (13-48) & $\begin{array}{l}\text { Cálculos } \\
\text { ureterais: } 150 \\
\text { dias (56-1280) } \\
\text { e cálculos } \\
\text { vesicais: } 4 \\
\text { meses a } 8 \text { anos }\end{array}$ & & \\
\hline Localização dos Cálculos & & $\begin{array}{l}\operatorname{Rim} 37,5 \% \\
\text { Ureter } 12,5 \% \\
\text { Bexiga } 50 \%\end{array}$ & $\begin{array}{l}\text { Rim 20\% } \\
\text { Bexiga 73,3\% } \\
\text { Múltiplos sítios 6,7\% }\end{array}$ & $\begin{array}{l}\text { Maioria renal; } \\
\text { múltiplos sítios } \\
\text { em } 43,75 \%\end{array}$ & $\begin{array}{l}\operatorname{Rim} 75 \% \\
\text { Ureter } 25 \%\end{array}$ & $\begin{array}{l}\text { Ureter } 75 \% \\
\text { Bexiga } 25 \%\end{array}$ & $\begin{array}{l}\text { Rins } 67 \% \\
\text { Ureter } 33 \%\end{array}$ & \\
\hline Tamanho médio (mm) & & & $12(3,4-40)$ & 8,3 & $17(7-47)$ & & & \\
\hline LECO $\psi$ & $27,3 \%$ & $50 \%$ & & & $41,7 \%$ & $11,1 \%$ & $2 \%$ & $36 \% *$ \\
\hline Nefrolitotomia percutânea & $27.3 \%$ & & & $6,25 \%$ & $25 \%$ & $33,3 \%$ & $37 \%$ & $28 \%$ * \\
\hline Ureterorenoscopia Flexível & $18,2 \%$ & $25 \%$ & & & $25 \%$ & & $14 \%$ & $36 \% *$ \\
\hline Nefrolitotomia aberta & & & & $12,5 \%$ & & $11,1 \%$ & & \\
\hline Cistoscopia & & $100 \%$ & $61,5 \%$ & & & $100 \%$ & & \\
\hline Eliminação espontânea & & $25 \%$ & $38,5 \%$ & $31,2 \%$ & $8,3 \%$ & $33,3 \%$ & & \\
\hline Nefrectomia & & & & & & $11,1 \%$ & & \\
\hline Alcalinização da urina & & & & $43,75 \%$ & & & & \\
\hline
\end{tabular}

$\psi$ Litotripisia Extra-Corpórea; $\gamma 12$ cálculos em 10 pacientes; *Além dos 12 casos de litíase inclui 2 casos de recorrência

JBT J Bras Transpl. 2005;8:440-445 
Os principais estudos sobre litíase renal após o transplante renal são apresentados na Tabela 1.

Na população geral, cerca de 70 a $85 \%$ dos cálculos são compostos por oxalato de cálcio, seguida de outros sais de cálcio, ácido úrico ( 2 a $18 \%)$, fosfato de amônio magnesiano ou estruvita $(3,9 \%)$ e de cistina (1 a 2\%). ${ }^{35,36}$ Os cálculos estão associados à infecção urinária em 3 a $15 \%$ dos casos. ${ }^{36}$

Nos pacientes transplantados renais, o perfil da composição dos cálculos difere da população geral, de modo que os cálculos de ácido úrico ou associados à infecção urinária são mais freqüentes ${ }^{15,26,27}$, podendo chegar a $50 \%$ dos $\operatorname{casos}^{27}$, já que a imunossupressão predispõe à infecção urinária, sendo relatada infecção por Proteus mirabilis em mais de $50 \%$ dos pacientes com litíase renal. ${ }^{26}$

Em outros trabalhos, a composição dos cálculos no transplante renal também tem sido relatada: ácido úrico $37,5 \%$, oxalato de cálcio $37,5 \%$ e fosfato de amônio magnesiano ( $25 \%) .{ }^{26}$ Contudo, a incidência de cálculo de oxato de cálcio pode ser maior após o transplante renal $(69 \%){ }^{16}$

As alterações metabólicas que se associam ao transplante renal incluem: hipocitratúria (75\%), hiperparatiroidismo (36\%), hipofosfatemia (24\%) e hipercalcemia $(10 \%) .{ }^{20}$ Por outro lado, há relatos de que, em $90 \%$ dos pacientes transplantados renais, não haveria distúrbios metabólicos relacionados ao maior risco de litíase renal. ${ }^{9}$

A ciclosporina pode levar à hiperuricemia, que é decorrente da redução da taxa de filtração glomerular, ${ }^{3}$ além de hiperuricosúria em 50 a $60 \%$ dos pacientes transplantados renais. ${ }^{38}$ Está associada também ao aumento da calciúria através da inibição da calbindina D-28k, assim como o tacrolimus. ${ }^{39-41}$ A calbindina D-28k é uma proteína intracelular ligadora do cálcio que é expressa exclusivamente no túbulo distal e que está associada à reabsorção do cálcio neste segmento do néfron. O sirolimus, por sua vez, não interfere no metabolismo do cálcio. ${ }^{41}$

O diagnóstico precoce, a remoção dos cálculos e as medidas preventivas podem reduzir os efeitos adversos na função do enxerto renal.

A função do enxerto renal pode se alterar ${ }^{17,22}$ ou não $0^{9,17,23-27}$ após o tratamento da litíase do rim transplantado.

O tratamento da litíase renal após o transplante depende do tamanho do cálculo, da sua localização e da experiência do centro transplantador. Os tratamentos realizados incluem: LECO em 11,1 a $87 \%^{9,16,17,19,21,23,24,26,30}$, litotripsia mecânica endoscópica em $21,7 \%{ }^{16}$, nefrolitotomia percutânea em 4,3 a $37 \%$ 9,15,16,17,21,24,26, nefrolitotomia aberta em 11,1 a $12,5 \%{ }^{15,17}$, ureterorenoscopia em 14 a 35,7\% 9,19,21,23,24,26 e observação clínica em 6,25 a 26,1\%. ${ }^{15,16} \mathrm{~A}$ cistoscopia tem sido realizada para remoção dos cálculos vesicais em 50 a $100 \%$ dos casos. . $^{17,19,22,23}$

Contudo, o tratamento da litíase no rim transplantado é similar àquele para rim único, de modo que para cálculos até $2 \mathrm{~cm}$, a LECO é sugerida. ${ }^{25,26} \mathrm{Se}$ o cálculo tiver entre $1 \mathrm{a} 2 \mathrm{~cm}$, recomenda-se o implante do cateter duplo J, enquanto que, para cálculos maiores do que $2 \mathrm{~cm}$, a nefrolitotomia percutânea é o procedimento mais indicado. ${ }^{25,26,42}$ A nefrolitotomia percutânea pode também ser realizada para remoção de cálculos coraliformes no rim transplantado. ${ }^{43,44} \mathrm{~A}$ eliminação espontânea do cálculo pode ocorrer em cerca de 8,3 a $40 \%$ dos pacientes. ${ }^{9} 15,16,19,22,23,29$

Apesar de menos invasiva, a LECO no rim transplantado tem algumas restrições: o acesso ao cálculo no rim transplantado pode ser difícil devido às estruturas ósseas; a eliminação dos fragmentos pode ser limitada, especialmente para cálculos no grupamento calicial inferior, que estão associados à alta taxa de fragmentos residuais ( 25 a 40\%); a hidrodinâmica normal pode estar alterada no rim transplantado devido à sua rotação quando implantado na fossa ilíaca; os fragmentos residuais dos cálculos fragmentados apresentam risco elevado de infecção urinária; e a eliminação de cálculos maiores que $2,5 \mathrm{~cm}$ é bastante limitada pela LECO ${ }^{13,45}$ Além disto, a permanência de fragmentos dos cálculos é assintomática, já que o ureter é denervado, o que aumenta ainda mais o risco de infecção urinária.

Para os cálculos ureterais, a ureteroscopia é tecnicamente difícil e muitas vezes sem sucesso ${ }^{(10)}$. Na anastomose uretero-vesical Leadbetter-Politano, a posição do neo-orifício oferece melhor acesso para o ureter através da ureteroscopia. Assim, a anastomose LeadbetterPolitano é recomendada para pacientes com risco elevado de formação de cálculos, após o transplante renal. De qualquer forma, preconiza-se a realização de ureteroscopia com aparelho flexível, já que a falta de suporte adequado no ureter transplantado e a fibrose peri-ureteral tornam a ureteroscopia rígida um procedimento arriscado. ${ }^{10,13}$

A nefrolitotomia percutânea é um procedimento bem tolerado e com baixo índice de complicações. ${ }^{13,46,47}$ É recomendada a realização de ultrassom para guiar a punção pélvica e evitar lesão intestinal. A dilatação percutânea pode apresentar certa dificuldade devido à fibrose peri-renal, que pode, por outro lado, ser uma vantagem, ao permitir a fixação do rim transplantado durante a dilatação. A remoção da nefrostomia não difere do rim não transplantado. ${ }^{13}$

A nefrolitotomia percutânea pode também ser realizada para cálculo coraliforme no rim transplantado de doador falecido e com anastomose uretero-intestinal Bricker, embora haja o risco de fístula urinária no orifício da nefrostomia devido a altos valores de pressão no trato urinário superior. ${ }^{43}$

Em relação à composição do cálculo e o tratamento, quando a origem é de cálcio, a LECO guiada por ultrassom é a melhor opção, enquanto que, para o cálculo de ácido úrico, dá-se preferência para o tratamento clínico com alcalinização para dissolução do cálculo com citrato ou bicarbonato. ${ }^{15,26,27}$

As recomendações na dieta são as mesmas para a população geral, incluindo aumento da ingestão de líquidos e restrição do consumo de sal, proteína e oxalato. ${ }^{26}$ Os pacientes que utilizam alopurinol devem ter a dose da azatioprina reduzida em $50 \%$ ou sua substituição pelo micofenolato, devido ao risco aumentado de desenvolver leucopenia. ${ }^{26}$

\section{ABSTRACT}

The incidence of renal lithiasis is increasing all over the world. It is a relative counter-indication to the kidney donation. Whenever a potential donor has a previous history of kidney lithiasis, the donation is allowed except upon the presence of metabolic disorder diagnosis, such as hypercalciuria, hyperuricosuria, hyperoxaluria, hypocitraturia or metabolic acidosis; cistine or struvite stones; urinary tract infection and multiple calculi or nephrocalcinosis. The calculi presented in kidney of deceased or living donors may spontaneously disappear, or they can be removed by ureteroscopy, percutaneous nephrolithotomy or extracorporeal shock wave lithotripsy. Renal lithiasis after kidney 
transplantation is not frequent, ranging from 0.2 to $3 \%$ (mean 1\%), and it can occur either de novo or be recurrent. Patients are usually asymptomatic, since the transplanted kidney is denervated. The diagnosis occurs from 3 months to 3 years after transplant, but it is most frequently diagnosed during the first year. The calculi composition is quite different from the general population, being more frequent the uric acid stone and those associated to the urinary tract infection. Treatment depends on the size and location of the calculi and the experience of the transplantation center, and it includes extracorporeal shock wave lithotripsy, open or percutaneous nephrolithotomy, ureterorenoscopy, cistoscopy, and clinical observation (spontaneous passage).

Keywords: Nephrolithiasis, Kidney Transplantation, Treatment, Organ Donors.

\section{REFERÊNCIAS}

1. Moe OW. Kidney stones: pathophysiology and medical management. Lancet. 2006; 367:333-44

2. Coe FL, Evan A, Worcester E. Kidney stone disease. J Clin Invest. 2005;111(10): 2598-608.

3. Palma D, Pozzi-Mucelli R, Stacul F. Present-day imaging of patients with renal colic. Eur Radiol. 2001;11(1):4-17

4. Otal P, Irsutti M, Chabbert V, Murat C, Duchase JL, Rousseau H, et al. Exploration radiologique de la colique néphrétique. J Radiol. 2001;82(1):27-33.

5. Capocasale E, Busi N, Mazzoni MP, Alessandri L, Sianesi M. Donor graft lithiasis in kidney transplantation. Transplant Proc. 2002;34:1191-2.

6. Worcester E, Parks JH, Josephson MA, Thisted RA, Coe FL. Causes and consequences of kidney loss in patients with nephrolithiasis. Kidney Int. 2003;64(6):2204-13.

7. Gabolde M, Herve C, Moulin AM. Evaluation, selection, and follow up of live kidney donors: a review of current practice in French renal transplant centers. Nephrol Dial Transplant. 2001;16:2048-52.

8. Monaco AP, Morris PJ. Care of the live kidney donor: consensus on the ultimate gift. Transplantation. 2005;79 (Suppl 2):S51-S66.

9. Klingler HC, Kramer G, Lodde M, Marberger M. Urolithiasis in allograft kidneys. Urology. 2002;59:344-8.

10. Devasia A, Chacko N, Gnanaraj L, Cherian R, Gopalakrishnan G. Stone-bearing live-donor kidneys for transplantation. BJU Int. 2005;95:394-7.

11. Rashid MG, Konnak JW, Wolf JS Jr, Punch JD, Magee JC, Arenas JD, et al. Ex vivo ureteroscopic treatment of calculi in donor kidneys at renal transplantation. J Urol. 2004;171(1):58-60

12. Yigit B, Aydin Ç, Titiz I, Berber I, Sinanoglu O, Altaca G. Stone disease in kidney transplantation. Transplant Proc. 2004;36:187-9.

13. Lu HF, Shekarriz B, Stoller ML. Donor-gifted allograft urolithiasis: early percutaneous management. Urology. 2002;59:25-7.

14. Qazi YA, Ali Y, Venuto RC. Donor calculi induced acute renal failure. Ren Fail. 2003;25(2):315-22.

15. Lancina Martin JA, Garcia Buitron JM, Diaz Bermudez J, Alvarez Castelo L, Duarte Novo J, Sanchez Herino JM. Urinary lithiasis in transplanted kidney. Arch Esp Urol. 1997;50(2):141-50

16. Torrecilla Ortiz C, Gonzalez-Satue C, Riera Canals L, Colom Feixas S, Franco Miranda E, Aguilo Lucia F, et al. Incidence and treatment of urinary lithiasis in renal transplantation. Actas Urol Esp. 2001;25(5):357-63.

17. Streeter EH, Little DM, Cranston DW, Morris PJ. The urological complications of renal transplantation: a series of 1535 patients. BJU Int. 2002;90:627-34.
18. Shoskes DA, Hanbury D, Cranston D, Morris PJ. Urological complications in 1000 consecutive renal transplant recipients. J Urol. 1995;153(1):18-21.

19. El-Mekresh M, Osman Y, Ali-El-Dein B, El-Diasty T, Ghoneim MA. Urological complications alter living-donor renal transplantation. BJU Int. 2001;87:295-306.

20. Harper JM, Samuell CT, Hallson PC, Wood SM, Mansell MA. Risk factors for calculus formation in patients with renal transplants. Br J Urol. 1994;74:147-50.

21. Abbott KC, Schenkman N, Swanson SJ, Agodoa LY. Hospitalized nephrolithiasis after renal transplantation in United States. Am J Transplantation. 2003;3:465-70.

22. Kim H, Cheigh JS, Ham HW. Urinary stones following renal transplantation. Korean J Intern Med. 2001;16:118-22.

23. Rhee BK, Bretan PN, Stoller ML. Urolithiasis in renal and combined pancreas/ renal transplant recipients. J Urol. 1999;161(5):1458-62.

24. Benoit G, Blanchet P, Eschwege P, Jardin A, Charpentier B. Occurrence and treatment of kidney graft lithiasis in a series of 1500 patients. Clin Transplant. 1996;10(2):176-80.

25. Rodrigo Aliaga M, Morera Martinez J, Lopez Alcina E, Broseta Rico E, Oliver Amoros F, Boronot Tormo F, et al. Lithiasis of the transplanted kidney: therapeutical potential. Arch Esp Urol. 1996;49(10):1063-70.

26. Doehn C, Fornara P, Tiemer C, Fricke L, Jocham D. Renal transplant lithiasis. Transplant Proc. 2002;34:2222-3.

27. Rubio Briones J, Chechile Toniolo G, Parada Moreno R, Minino Pimentel L, Garcia Penit J, Caparros Sariol J, et al. Lithiasis in renal transplantation. Actas Urol Esp. 1995;19(7):561-5.

28. Santiago-Delpin EA, Baquero A, Gonzalez Z. Low incidente of urologic complications after renal transplantation. Am J Surg. 1986;151(3):374-7.

29. Hayes JM, Streem SB, Graneto D, Hodge EE, Steinmuller DR, Novick AC. Renal transplant calculi. A reevaluation of risks and management. Transplantation. 1989; 47(6):949-52.

30. Millan Rodriguez F, Gonzalez de Chaves E, Rousand Baron F, Izquierdo Latorre F, Rousand Baron A. Treatment of urinary calculi in transplanted kidney with extracorporeal shock wave lithotripsy. Arch Esp Urol. 2003;56(7):793-8.

31. Cologna AJ, Martins ACP, Suaid HJ, Tucci S, dos Reis RB, Paschoal RM, et al. Urolitíase no alotransplante renal. Acta Cir Bras. 2003;18(suppl 5):43-44.

32. Cho DK, Zackson DA, Cheigh J, Stubenbord WT, Stenzel KH. Urinary calculi in renal transplant recipients. Transplantation.1988;45(5):899-902.

33. Crook TJ, Keoghane SR. Renal transplant lithiasis: rare but time-consuming. BJU Int. 2005;95:931-3.

34. Dumoulin G, Hory B, Nguyen NU, Henriet MT, Bresson C, Bittard H, et al. Lack of increased urinary calcium-oxalate supersaturation in long-term kidney transplant recipients. Kidney Int. 1997;51(3):804-10. 
35. Gault MH, Chafe L. Relationship of frequency, age, sex, stone weight and composition in 15624 stones: comparison of results for 1980 to 1983 and 1995 to 1998. J Urol. 2000;164(2):302-7.

36. Teselius HG. Metabolic evaluation of patients with stone disease. Urol Int 1997;59 (3);131-41.

37. Hansen JM, Fogh-Andersen N, Leyssac PP, Strandgaard S. Glomerular and tubular function in renal transplant patients treated with and without ciclosporin A. Nephron. 1998;80(4):450-7.

38. Norlen BJ, Hellstrom M, Nisa M, Robertson WG. Uric acid stone formation in a patient with kidney transplantation: metabolic considerations. Scan J Uro Nephrol. 1995;29:335-7.

39. Lee CT, Huynh VM, Lai LW, Lien YH. Cyclosporine A-induced hypercalciuria in calbindin-D28K knockout and wild-type mice. Kidney Int. 2002;62:2055-61.

40. Yang CW, Kim J, Kim YH, Cha JH, Mim SY, Kim YO, et al. Inhibition of calbindin D28K expression by cyclosporin A in rat kidneys: the possible pathogenesis of cyclosporin A-induced hypercalciuria. J Am Soc Nephrol. 1998;9(8):1416-26.
41. Aicher L, Meier G, Norcross AJ, Jakubowski J, Varela MC, Cordier A, et al. Decrease in kidney calbindina D-28k as a possible mechanism mediating cyclosporine A- and FK-506induced calciuria and tubular mineralization. Biochem Pharmacol. 1997;53(5):723-31.

42. Wheatley M, OhlDA, Sonda LP, Wang SC, Konnak JW. Treatment of transplant stones by extracorporeal shock wave lithotripsy in the prone position. Urology. 1991;37:57-60.

43. Tanneau Y, Vidart A, Sibert L, Grise P, Pfister C. Management of coralliform lithiasis on renal allograft with bricker-type ureterointestinal anastomosis. Transplant Proc. 2005;37(5):2104-6.

44. Francesca F, Felipetto R, Mosca F, Boggi U, Rizzo G, Puccini R. Percutaneous nephrolithotomy of transplanted kidney. J Endourol. 2002;16(4):225-7.

45. Bhadauria RP, Ahlawat R, Kumar RV, Srinadh ES, Banerjee GK, Bhandari M. Donor-gifted allograft lithiasis: extracorporeal shock wave lithotripsy with over table module using the Lithostar Plus. Urol Int. 1995;55:51-5.

46. Citterio F, Grassetti F, Nanni G, Azzaretto M, Avolio AW, Castagneto M. Accidental transplantation of a kidney with stones: case report. Transplant Proc. 1991;23:2560.

47. Caldwell TC, Burns JR. Current operative management of urinary calculi after renal transplantation. J Urol. 1998;140(6):1360-3. 